\title{
NOTE ON CONNECTEDNESS IN TOPOLOGICAL RINGS
}

\author{
J. GIL DE LAMADRID AND J. P. JANS
}

We consider certain properties of topological rings with identity which can be deduced from connectedness.

The following two statements follow immediately from Kaplansky [2, Theorems 1 and 2].

1. A connected compact ring is a zero ring.

2. A connected locally compact ring with identity is a finite dimensional algebra over the reals. In this note we drop the assumption of local compactness. Most of the results are consequences of the following simple lemma.

Let $M$ be a topological module over a topological ring $A$, i.e. $M$ is a topological abelian group, a module over $A$, and $(a, m) \rightarrow a m$ is jointly continuous.

Lemma 1. If $A$ is connected and $M_{0}$ is a submodule of $M$ then $A M_{0}$ is in the component of 0 in $M_{0}$.

Proof. Let $m_{0} \in M_{0}$, then $a \rightarrow a m_{0}$ is a continuous mapping of $A$ onto a connected subset of $M_{0}$. Since for $a=0$ in $A, a m_{0}=0$, this subset contains 0 .

The following statements follow immediately from Lemma 1:

1. A unital module over a connected ring with identity has only connected submodules.

2. A connected ring with identity has only connected left (right) ideals.

3. The only discrete left (right) ideal in a connected ring with identity is the zero ideal.

We use the definition of covering space and simple connectedness given by Chevalley [1], and we prove the following theorem which is in direct analogy to Proposition 5, p. 53 of [1].

TheOREM 1. If a ring $A$ admits a simply connected covering space $(S, f)$ then $S$ can be made into a ring so that $f$ is a ring homomorphism; furthermore if $A$ has an identity so does $S$.

Proof. Assume that $(S, f)$ covers $A$ and that $S$ is simply connected. Then the product $T=S \times S \times S \times S$ is also simply connected. Define the continuous mapping $\Omega: T \rightarrow A$ by $\Omega(a, b, c, d)=f(a) f(b)$ $+f(c)-f(d)$. Let 0 be an element of $S$ contained in $f^{-1}(0)$, and let $\beta$ be a fixed element of $S$ not contained in $f^{-1}(0)$. The simple connected-

Received by the editors August 17, 1956. 
ness of $T$ then implies there is a unique "lifting" of $\Omega$ (a continuous mapping of $\Omega^{\prime}: T \rightarrow S$ such that $\left.f \circ \Omega^{\prime}=\Omega\right)$ such that $\Omega^{\prime}(\beta, 0,0,0)=0$. We define $-a$ in $S$ to be $\Omega^{\prime}(\beta, 0,0, a)$ and $a+b$ to be $\Omega^{\prime}(\beta, 0, a,-b)$. These are continuous operations, $S$ becomes an abelian topological group under them, and $f$ is a group homomorphism of $S$ onto the additive group of $A$ (see Proposition 5, p. 53 of [1]).

We now define a (continuous) multiplication in $S$ by $a b$ $=\Omega^{\prime}(a, b, 0,0)$. Note that $f(a b)=f(a) f(b)$.

We show first that $a 0=0 a=0$ for all $a$ in $S$. The two continuous mappings $\theta: a \rightarrow 0$ and $\theta^{\prime}: a \rightarrow a 0$ have the property that $f \circ \theta=f \circ \theta^{\prime}$ and $\theta(\beta)=\theta^{\prime}(\beta)$. Thus since $S$ is connected and $f$ is a covering map $\theta=\theta^{\prime}, a 0=0$. Also the two mappings $\theta$ and $\theta^{\prime \prime}: a \rightarrow 0 a$ obey $f \circ \theta=f \circ \theta^{\prime \prime}$ and agree on $a=0$, thus $\theta=\theta^{\prime \prime}$ and $0 a=0$. We next show associativity.

The two continuous mappings $\Sigma:(a, b, c) \rightarrow a(b c)$ and $\Sigma^{\prime \prime}:(a, b, c)$ $\rightarrow(a b) c$ from the connected space $S \times S \times S$ to $S$ have the property that $f \circ \Sigma=f \circ \Sigma^{\prime \prime}$ and $\Sigma$ and $\Sigma^{\prime \prime}$ agree on $(0,0,0)$. Thus $\Sigma=\Sigma^{\prime \prime}$ and $a(b c)=(a b) c$. To show left distributivity use the above argument applied to the two mappings $(a, b, c) \rightarrow a(b+c)$ and $(a, b, c) \rightarrow a b+a c$ and similarly for right distributivity.

If $A$ has an identity $e$ then the element $\beta$ may be chosen in $f^{-1}(e)$ and is an identity for $S$. This is because the two mappings $b \rightarrow \beta b$ and $b \rightarrow b$ have the same composition with $f$ and agree on $b=0$. A similar argument shows $b \beta=b$. This completes the proof of the theorem.

As an immediate corollary we obtain

THEOREM 2. A ring with identity which admits a simply connected covering space is already simply connected.

Proof. By Theorem 1, the ring admits a covering ring with identity and the covering map becomes a ring homomorphism. But the kernel of this is a discrete ideal and, by the property 3 listed above, must be zero. Thus the covering map is a homeomorphism.

\section{REFERENCES}

1. C. Chevalley, Theory of Lie groups, Princeton University Press, 1946.

2. I. Kaplansky, Locally compact rings I, Amer. J. Math. vol. 70 (1948) pp. 447459.

The Ohio State University 